\title{
C-Fos Expression in the Spinal Cord and Pain-related Symptoms Induced by Chronic Arthritis in the Rat Are Prevented by Pretreatment with Freund Adjuvant
}

\author{
Catherine Abbadie, Jean-Marie Besson, and Bernard Calvino ${ }^{a}$ \\ Physiopharmacologie du Système Nerveux, INSERM U161, and Physiopharmacologie de la Douleur EPHE, 75014 Paris, \\ France
}

We previously showed that pathological adjuvant-induced arthritis (AIA), a chronic pain model, is associated with an increase in the number of Fos-like immunoreactivity (FosLI) in lumbar spinal cord neurons; maximal Fos-LI corresponds to the peak of pathological and behavioral signs of the disease. On the other hand, AIA is prevented in rats that have previously been injected with diluted complete Freund adjuvant (CFA). Here, the effects of pretreatment with CFA were studied in parallel on both pathological parameters and pain-related tests, and on Fos-LI, 3 weeks after the inoculation of the arthritogenic solution during the acute phase of the disease, that is, when hyperalgesia is maximal. One group of rats (vaccinated group) was pretreated with diluted CFA, 3 weeks and 1 week before inoculation (AIA) (with the concentrated arthritogenic solution), while a second group (arthritic group) was pretreated with the vehicle for the CFA solution and then inoculated with the concentrated arthritogenic solution. Control groups consisted of rats treated with various combinations of either saline or vehicle solutions during the pretreatment periods or at the time of inoculation.

The arthritic group developed all pathological symptoms of AIA (i.e., loss of weight, difficulties in walking, increases in paw diameter, and decreases in the vocalization thresholds to mechanical pressure of the hindpaw, and finally an increase in the vocalization response to flexion and extension). In contrast, the vaccinated group did not develop AIA symptoms. The total number of Fos-LI neurons in the L3-L4 segments of the spinal cord, located mainly in the neck of the dorsal horn (laminae $V$ and VI) and in the ventral horn, was high in the arthritic group but was significantly decreased $(68 \%$ decrease, $p<0.001)$ in the vaccinated group. Importantly, the pathological parameters of arthritis correlated with the number of Fos-LI neurons in the L3-L4 segments. The pathological parameters and the pain-related test scores did not significantly differ in the vaccinated group as compared to the four control groups.

This study demonstrates that the increase in the number of Fos-LI neurons during arthritis that could indirectly reflect

\footnotetext{
Received Nov. 24, 1994; revised Mar. 21, 1994; accepted Apr. 7, 1994.

We gratefully acknowledge Dr. A. H. Dickenson for English revision of the manuscript. This study was supported by INSERM funds and by an unrestricted grant from Bristol-Myers Squibb.

Correspondence should be addressed to Catherine Abbadie, INSERM U161, 2 rue d'Alèsia, 75014 Paris, France.

aPresent address: INSERM CJF 91-02 Faculté de Médecine, 8 rue du généra Sarrail, 94010 Créteil Cédex.

Copyright (C) 1994 Society for Neuroscience $0270-6474 / 94 / 145865-07 \$ 05.00 / 0$
}

spontaneous chronic pain and hyperalgesia was prevented by pretreatment with diluted $F A$, which protected rats against the development of AIA; the marked decrease in Fos expression was in good agreement with behavioral and pathological data.

IKey words: c-Fos, immediate-early gene, adjuvant-induced arthritis, spinal cord, nociception, adjuvant pretreatment]

Hunt et al. (1987) demonstrated that physiological stimulation of rat primary sensory neurons causes the expression of Foslike immunoreactivity (Fos-LI) in nuclei of postsynaptic neurons of the dorsal horn of the spinal cord. This work has led to numerous studies related to pain research. After several years of investigations, there is accumulating evidence that various nociceptive peripheral stimuli result in c-fos expression at the spinal cord level (Hunt et al., 1987; Bullitt, 1989, 1990, 1991; Draisci and Iadarola, 1989; Menétrey et al., 1989; Williams et al., 1989; Presley et al., 1990; Tölle et al., 1990; Wisden et al., 1990; Gogas et al., 1991; Herdegen et al., 1991; Naranjo et al., 1991; Abbadie and Besson, 1992a,b; Bullitt et al., 1992; Hammond et al., 1992; Hylden et al., 1992; Leah et al., 1992; Lee and Beitz, 1992; Noguchi et al., 1992; Abbadie and Besson, 1993). Taking into account the numerous investigations based on c-Fos experiments, it is reasonable to assume that dorsal horn neurons receiving noxious inputs (sec references in Bcsson and Chaouch, 1987) preferentially express c-fos when activated by noxious stimulation. This is strongly supported by the fact that electrical stimulation of the sciatic nerve at $\mathrm{A} \delta / \mathrm{C}$ intensity, but not at $A \alpha / \beta$, induced c-fos expression in lumbar spinal cord neurons (Herdegen et al., 1991).

C-fos expression in spinal cord neurons occurs does not only after acute stimulation and inflammation, but also in chronic pain diseases such as poly- or monoarthritis (Abbadie and Besson, 1992a; Lantéri-Minet et al., 1992). We previously demonstrated that the number of Fos-LI neurons in lumbar spinal cord follows in parallel the time course of adjuvant-induced arthritis disease (AIA; triggered by the injection of heat-killed Mycobacterium butyricum, i.e., complete Freund adjuvant, CFA; Pearson and Wood, 1959; Pearson et al., 1961). In contrast to acute inflammation (Draisci and Iadarola, 1989; Menétrey et al., 1989; Noguchi et al., 1991), which mainly induces c-fos expression in the superficial dorsal horn, polyarthritis induces c-fos expression in deep laminae of the dorsal horn and in the ventral horn (Abbadie and Besson, 1992a). Briefly, the peak of the distribution of Fos-LI neurons during the development of polyarthritis corresponds to the maximum hyperalgesia, that is, 3 weeks after inoculation of the disease (see references in Besson 
Table 1. Pathological observations and the number of Fos-like-immunoreactive (Fos-LI) neurons in arthritic, vaccinated and control rats

\begin{tabular}{lllll} 
Groups & Weight & Mobility score & Ankle diameter & Fos-LI neurons \\
\hline Polyarthritic & $423 \pm 16$ & $2.1 \pm 0.5$ & $8.9+0.8$ & $42.5 \pm 5.0$ \\
Vaccinated & $464 \pm 12$ & $3.9 \pm 0.1$ & $6.8 \pm 0.1$ & $13.5 \pm 5.2$ \\
Control 1 & $480 \pm 11$ & $4.0 \pm 0.0$ & $6.4 \pm 0.2$ & $14.9 \pm 5.4$ \\
Control 2 & $504 \pm 12$ & $4.0 \pm 0.0$ & $6.5 \pm 0.1$ & $17.1 \pm 4.2$ \\
Control 3 & $490 \pm 13$ & $4.0 \pm 0.0$ & $6.3 \pm 0.1$ & $13.7 \pm 3.1$ \\
Control 4 & $476 \pm 19$ & $4.0 \pm 0.0$ & $6.5 \pm 0.0$ & $3.3 \pm 2.0$ \\
$F(5,32)=$ & $4.5, p<0.004$ & $10.4, p<0.0001$ & $6.4, p<0.0003$ & $6.9, p<0.0002$ \\
$R^{2}$ & $0.25, p<0.002$ & $0.53, p<0.0001$ & $0.44, p<0.0001$ & -
\end{tabular}

For the details of the groups of animals, see Materials and Methods. The weight is expressed in grams, the mobility scale is from 0 to 4 (see Material and Methods), and the paw diameter is the mean of the two ankles, expressed in mm. The numbers of Fos-LI neurons were counted in all laminae of 20 sections from L3-LA segments and is expressed as neurons per section. The penultimate row gives the one-way ANOVA in the six groups. The last row gives the $R^{2}$ values and the significance for each preclinical parameter as compared to the number of Fos-LI neurons. Note that for the weight, the mobility score, the paw diameter, and the number of Fos-LI neurons, the vaccinated group and the four control groups significantly $(p<0.05$ at least) differ from the arthritic group.

and Guilbaud, 1988). It has previously been shown that rats that have recovered from arthritis are resistant to a subsequent injection of the arthritogenic solution (Waksman et al., 1960; Wood and Pearson, 1962). More recently it has been shown that pretreatment with diluted CFA strongly decreases not only the inflammatory pathological signs of the disease, but also the pain-rclated behaviors (Calvino et al., 1991a). In order to extend the correlation between Fos-LI in lumbar spinal cord neurons and the development of polyarthritis, the aim of the present study was to gauge if a pretreatment with a diluted Mycobacterium butyricum solution, which is known to prevent AIA (Gery and Waksman, 1967), will also prevent c-fos expression.

In the present experiment we considered at the maximal stage of the disease ( 3 weeks) the effects of a pretreatment with diluted CFA by measuring in parallel Fos-LI in the lumbar spinal cord and the pathological - that is, body weight, diameter of ankles, and mobility score-and behavioral-that is, vocalization threshold evoked by calibrated paw pressure applied on an inflamed hindpaw, and vocalization evoked by flexion and extension of an inflamed ankle-signs of polyarthritis.

A preliminary report has briefly described some of these data (Calvino et al., 1993).

\section{Materials and Methods}

Experimental animals. Experiments were performed on 74 male Sprague-Dawley rats (Charles River, France), weighing 200-225 gm at the beginning of the experimental procedure. As previously described by Calvino et al. (1991a), pretreatment consisted of two subcutaneous injections with a $50 \%$ commercial diluted CFA solution [1 $\mathrm{mg}$ of killed and dried Mycobacterium butyricum in saline; Sigma; initially $100 \mu \mathrm{l}$ in the two forepaws, and two subsequent subcutaneous injections of $100 \mu \mathrm{l}$ in the base of the dorsal part of the neck. The first pretreatment was made 3 weeks before induction of polyarthritis, and the second, 1 week before it. Polyarthritis was induced by injecting in both hindpaws with $50 \mu 1$ of the concentrated arthritogenic solution, that is, CFA (6 mg of Mycobacterium butyricum; Difco Laboratory) suspended in $1 \mathrm{ml}$ of an emulsion of liquid paraffin/saline/Tween $80,6: 4: 1$ by volume; solution was sterilized $20 \mathrm{~min}$ at $120^{\circ} \mathrm{C}$ in an autoclave].

Six groups of animals were used, two experimental groups and four control groups.

The two experimental groups consisted of (1) rats pretreated with the diluted CFA solution containing the bacteria and then inoculated with the concentrated arthritogenic solution (vaccinated group, $n=7$ ), and (2) rats pretreated with the same solution but without the bacteria, and then inoculated with the concentrated arthritogenic solution (arthritic group, $n=7$ ).
In order to detect any Fos-LI nuclei that was due to the repeated injections of the vehicle of the arthritogenic solution, rather than a consequence of the chronic disease, further control experimental groups consisted of rats that received different combinations of either saline or the vehicle (not containing the bacteria) of arthritogenic solution, as follows [control: pretreatment (neck, forepaws), inoculation (hindpaws)]: control $1(n=8)$ : vehicle, vehicle; control $2(n=8)$ : saline, vehicle; control $3(n=4)$ : vehicle, saline; control $4(n=4)$ : saline, saline.

The guidelines on ethical standards for investigations of experimental pain in animals were followed (Zimmermann, 1983). The number of experimental arthritic animals was kept to a minimum. They were housed three per large cage, the floor of which was covered with sawdust. They were kept in an animal room at a constant temperature of $22^{\circ} \mathrm{C}$, with a $12 \mathrm{hr}$ alternating light/dark cycle. Food and water were available ad libitum; the food was made available on the sawdust in the cages to minimize the need for the animals to make potentially painful movements to obtain food.

Pathological observations. In order to assess the development of the disease, we considered three pathological parameters 3 weeks after inoculation of AIA (i.e., when hyperalgesia is maximal) and on the day when animals were sacrificed. We took into account the weight of animals, the diameters of the ankles (mean of the two joints), and the mobility score as described by Butler et al. (1985), in which a five-level mobility scale was used: 0 , the rat lies down only; 1 , the rat crawls; 2 , the rat walks with difficulty; 3 , the rat walks and runs with difficulty; 4 , the rat walks and runs normally. In this set of animals, no behavioral test was performed, since stimulation of the animal induces c-fos expression. Since the study of Fos-LI and the assessment of pathological parameters were done in the same animals, there was the same number of animals per group as mentioned above and, moreover, it was possible to establish any correlation between pathological parameters and FosLI.

Pain-related tests. In a group of rats other than those used for FosLI studies, pain-related tests were performed (nine rats in each group). Three weeks after AIA inoculation, hyperalgesia developed; that is, manipulation of the hindpaws elicited a struggle response and vocalization. This hyperalgesia was quantitatively measured using two tests. (1) The "foot-bend" procedure (Kuzuna and Kawai, 1975; Winter et al., 1979) involves holding the rat comfortably and gently extending the left hindpaw. In these conditions a squeak could be elicited. Five stimuli were repeated at $5 \mathrm{sec}$ intervals and a rating of 0 or 1 was given if the animal emitted a squeak or not, respectively. Thus, for cach animal the rating ranged from 0 to 5 . In the second part of test, the left hindpaw was gently flexed five times at $5 \mathrm{sec}$ intervals, and an identical rating was used. The animal was then allowed to rest for $15 \mathrm{~min}$ in the cage and the second test was performed. (2) The "Randall-Selitto" test (1957) determines the threshold for vocalization induced by increasing calibrated pressure on one hindpaw, and then on the other hindpaw with a 5 min interval. The mean of the two determinations was calculated.

For the pathological observations and pain-related tests, statistical analysis was made to compare the different groups of animals, using one-way analysis of variance, and then for multiple comparisons, the 
PLSD Fisher's test was used. The investigator responsible for the measures was blind to the treatment of each animal.

Immunohistochemistry. Animals were deeply anesthetized with pentobarbital $(55 \mathrm{mg} / \mathrm{kg}$, i.p.) and perfused intracardially with $200 \mathrm{ml}$ of phosphate-buffered saline $0.1 \mathrm{M}$ followed by $500 \mathrm{ml}$ of $4 \%$ paraformaldehyde in $0.1 \mathrm{~m}$ phosphate buffer. The lumbar spinal cord was then removed and postfixed for $4 \mathrm{hr}$ in the same fixative and cryoprotected overnight in $30 \%$ sucrose in PB. Frontal frozen sections of $40 \mu \mathrm{m}$ were cut and collected in PB to be processed immunocytochemically as freefloating sections.

The serial sections were immunostained for c-fos-like protein according to the avidin-biotin-peroxidase method of Hsu et al. (1981). The tissue sections were immunoprocessed with the primary antiserum directed against the c-fos protein (OA 11-823, Cambridge Research Biochemicals). This Fos antibody is a sheep polyclonal antibody directed against residues $2-16$ of the $N$-terminal region of the molecule; it was used at 1:2000. For details of the procedure, see Abbadie and Besson (1993).

To test the specificity of the primary antibody, controls were performed; preabsorption with the corresponding synthetic peptide (OP11-3210, Cambridge Research Biochemicals) or omission of any stage. in the protocol abolished the staining.

Counting of Fos-labeled cells. Fos-LI was studied over two lumbar spinal levels, the L3 and L4 segments, in which the number of Fos-LJ neurons are the greatest in polyarthritic rats (Abbadie and Besson, 1992a). Tissue sections were first examined using dark-field microscopy to determine the segmental level according to Molander et al. (1984), and the gray matter landmarks. The sections were then examined under light-field microscopy to localize Fos positive cells. Labeled nuclei were counted using a camera lucida attachment. For each rat, two values were made: (1) the total number of Fos-LI neurons in the gray matter for 20 sections through L3-L4 segments, and (2) the number of Fos-LI neurons per specific laminar region of the spinal gray matter in these 20 sections. Four regions were defined: superficial dorsal horn (laminae I and II), nucleus proprius (laminae III and IV), neck and base of the dorsal horn (laminae V and VI), and the ventral medial gray (laminae VII, VIII, IX, and X; ventral). All results are expressed as the mean number of Fos-LI neurons per section. Statistical analysis was made to compare the numbers of labeled cells, using one-way analysis of variance for the total number in the whole gray matter, in the different groups of animals, two-way analysis of variance for the different groups of animals and the region. For multiple comparisons, Fisher's PLSD test was used. The investigator responsible for plotting and counting the Fos-LI neurons was blind to the treatment of each animal.

\section{Results}

\section{Clinical parameters}

No pain-related test was performed on the same set of animals in which Fos-LI study was performed, since acute peripheral stimulation of the animal will induce c-fos expression in spinal cord neurons. We thus considered three "pathological" parameters: the weight, the ability to move, and the diameter of the ankle. For all of the three parameters, the polyarthritic group exhibited clear pathological signs of polyarthritic disease, that is, loss of weight, difficulties in walking, and increases in paw diameter as compared to either the vaccinated group or to the four control groups; for all of the three parameters, the arthritic group significantly differed from the other groups $(p<0.05$ at least; Table 1). In addition, the pathological parameters of the vaccinated group did not significantly differ from those of the control groups (Table 1).

\section{Pain-related tests}

For the "foot bend" procedure, that is, flexion and extension, scores of the arthritic group were significantly greater as compared to the other groups ( $p<0.0001$ for the vaccinated and all the control groups as compared to the arthritic group). Similarly, there was a significant decrease in the threshold for vocalization induced by pressure applied to the foot in the arthritic
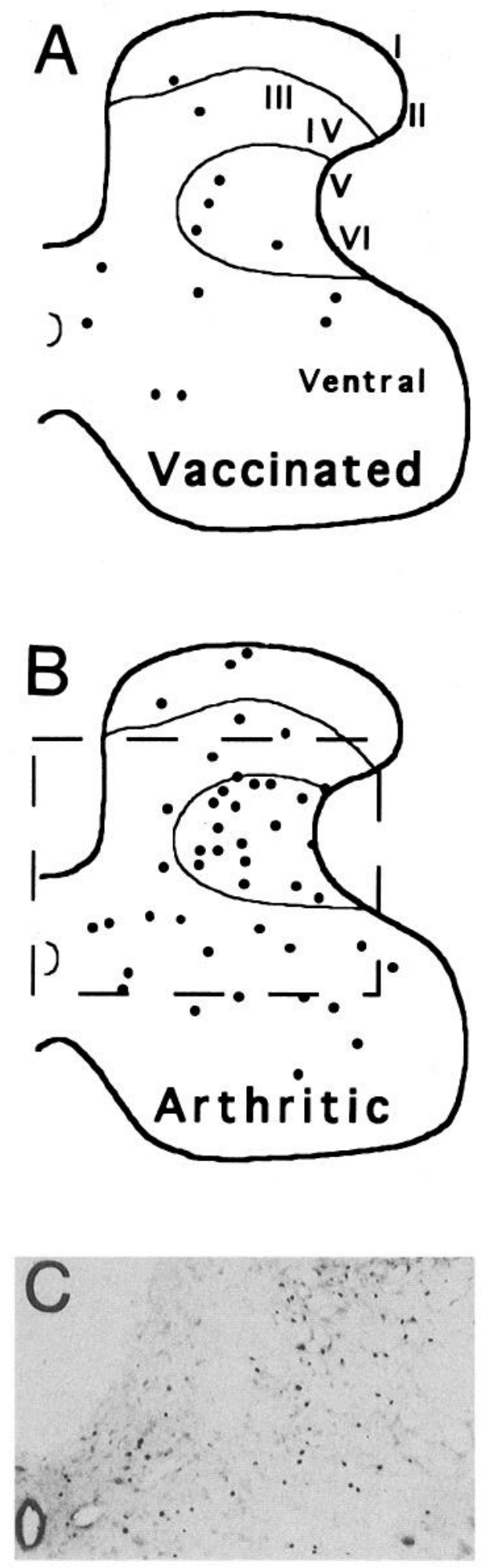

Figure 1. Camera lucida drawings and photomicrograph illustrating the location of Fos-like-immunoreactive (Fos-LI) neurons in the L4 segment of vaccinated $(A)$ and arthritic $(B, C)$ rats. The boundaries of the superficial laminae and of the reticular part of the neck of the dorsal horn are outlined for orientation. Note that (1) in the arthritic rat, intense labeling is observed in laminae V and VI and in the ventral horn and (2) in the vaccinated rat, considerably fewer cells are labeled. 


\begin{tabular}{lccl}
\hline $\begin{array}{l}\text { Table 2. } \\
\text { rats }\end{array}$ & Pain-related test scores in arthritic, vaccinated, and control \\
& & & \\
Groups & Flexion & Extension & $\begin{array}{l}\text { Randall- } \\
\text { Selitto }\end{array}$ \\
\hline Arthritic & $3.9 \pm 0.4$ & $2.1 \pm 0.5$ & $6.5 \pm 0.4$ \\
Vaccinated & $0.7 \pm 0.3$ & $0 \pm 0$ & $9.1 \pm 0.8$ \\
Control 1 & $0 \pm 0$ & $0 \pm 0$ & $9.3 \pm 0.6$ \\
Control 2 & $0 \pm 0$ & $0 \pm 0$ & $8.9 \pm 0.4$ \\
Control 3 & $0 \pm 0$ & $0 \pm 0$ & $9.1 \pm 0.4$ \\
Control 4 & $0 \pm 0$ & $0 \pm 0$ & $8.9 \pm 0.6$ \\
$F(5,48)=$ & $50.1, p<0.0001$ & $10.6, p<0.0001$ & $3.9, p<0.005$
\end{tabular}

For the details of the groups of animals and for the scores, see Material and Methods. The last row gives the one-way analysis of variance in the six groups. Note that for the "foot-bend" procedure (flexion and extension) and for the Randall-Selitto test, the scores of the arthritic group were significantly greater as compared to the other groups $(p<0.0001)$.

group as compared to the vaccinated and to the control groups (Table 2).

\section{Fos-like immunoreactivity}

Fos-LI nuclei appeared as round structures, stained to a greater or lesser extent (see Fig. 1), but to quantify the number of FosLI nuclei, we took into account all labeled nuclei without considering the intensity of the staining.

As we previously described, in polyarthritic rats, Fos-LI neurons were most numerous 3 weeks after the inoculation of the disease. They are present in all the lumbar enlargement and are most numerous in the L3-L4 segments (Abbadie and Besson, 1993). Thus, the quantification was only made in these segments.

As shown in Figures 1 and 2, Fos-LI was mainly distributed in the neck of the dorsal horn (laminae V and VI; $57 \%$, percentage vs all the laminae) and in the ventral horn (35\%), whereas very few Fos-LI neurons were observed in the superficial dorsal horn (laminae I and II; 3\%) and in the nucleus proprius (laminae III and IV; 5\%).

In vaccinated animals, that is, in animals pretreated with diluted Freund adjuvant, the number of Fos-LI neurons was three times less (see Table 1, Figs. 1,2) than in polyarthritic rats. In vaccinated animals and in the four groups of control animals the number of Fos-LI neurons was significantly $(p<$ $0.005)$ lower than in polyarthritic animals; considering the number of Fos-LI neurons in all the laminae $[F(5,32)=6.9, p<$ $0.0002]$ or in the neck of the dorsal horn $[F(5,32)=9.1, p<$ $0.0001]$ or in the ventral horn $[F(5,32)=8.5, p<0.0001]$. The number of Fos-LI neurons did not significantly differ between the vaccinated group and the four groups of control. In addition, there was no significant difference between the four control groups, although there was only a small number of labeled cells $(3.3 \pm 2.0$ neurons/section) in the group receiving only saline (control 4) as compared to the other control groups (mean of the three groups, 15 neurons/section).

\section{Correlation between Fos-like immunoreactivity results and clinical results}

Linear regression analysis showed that all of the three pathological parameters were significantly correlated with the total number of Fos-LI neurons in all laminae of L3-L4 segments (Table 1). The $R^{2}$ values and the significance for each parameter are given in Table 1. Thus, the pathological signs of arthritis,
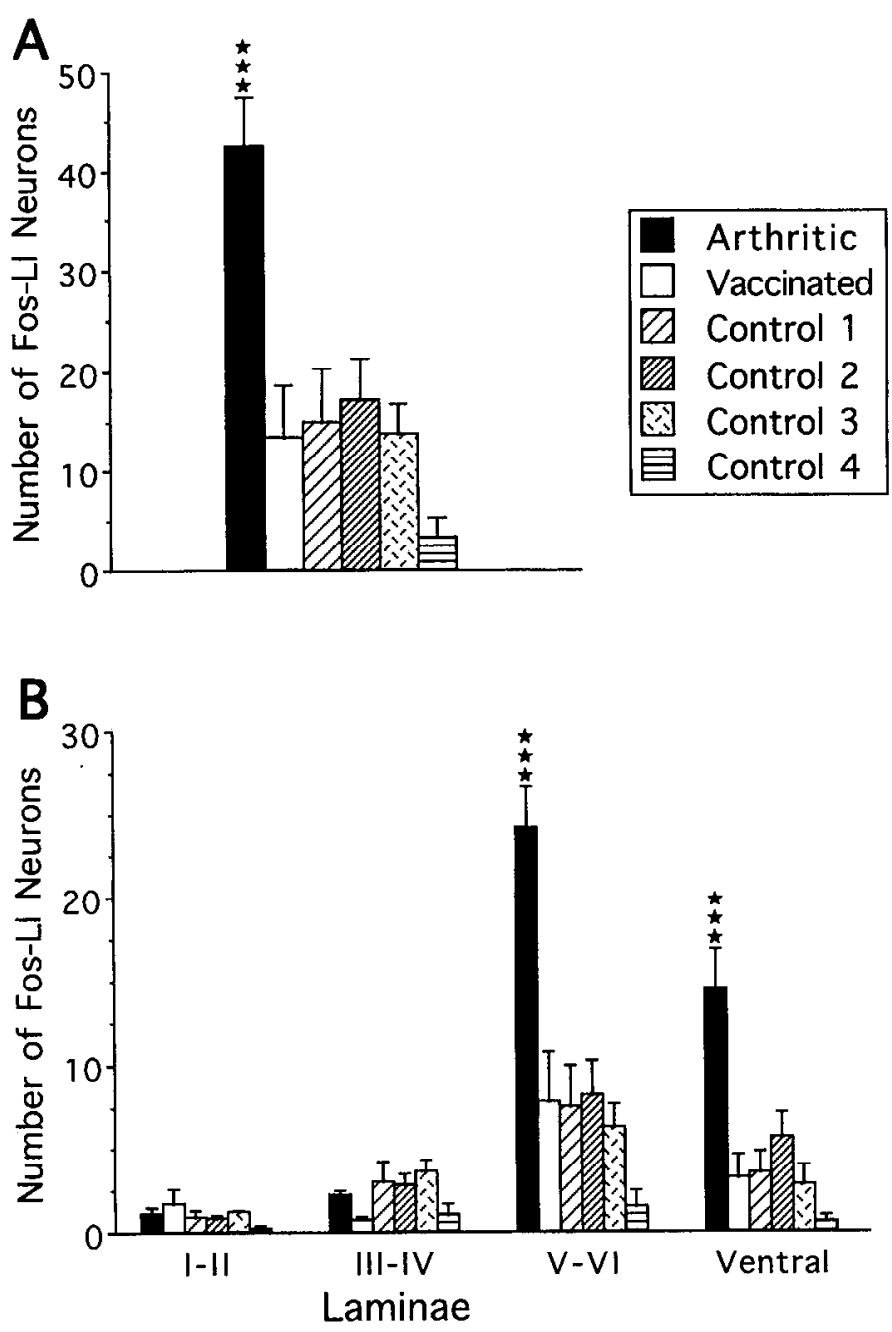

Figure 2. Histograms showing the number of Fos-like-immunoreactive $(F O S-L I)$ neurons in polyarthritic, vaccinated, and control rats. For details of the groups of animals sce Material and Methods. Results are expressed as mean (+SEM) number of Fos-LI neurons per section. $A$, Total number of Fos-LI neurons in all laminae of L3-L4 segments. $B$, Number of Fos-LI neurons per laminar region. Note that there is a significantly higher $(\star \star \star, p<0.001)$ number of Fos-LI neurons in the arthritic group as compared to the vaccinated group and the four control groups, considering either the total number of Fos-LI neurons, or the number of Fos-LI neurons, in laminae V and VI or in the ventral horn.

that is, loss of weight, difficulties in walking, and increase in paw diameter, were associated with a high number of Fos-LI neurons.

\section{Discussion}

In the present study, we showed that pretreatment with diluted CFA solution prevented both Fos-LI induced by AIA and the pathological and behavioral consequences of this chronic pain disease.

We previously found that the number of Fos-LI neurons in the lumbar spinal cord of arthritic rats studied throughout the development of AIA (Abbadie and Besson, 1992a) is in good agreement with pathological and behavioral studies describing different stages of this disease (Colpaert et al., 1980, 1987; De Castro Costa et al., 1981; Calvino et al., 1987). In fact, we observed (1) no significant labeling 1 week after AIA inocula- 
tion, which corresponds to the stage when the pathological signs and the behavioral modifications have not yet appeared; (2) a marked increase in the number of Fos-LI neurons 2 weeks after the inoculation and particularly after 3 weeks, which corresponds to the acute stage, that is, lack of mobility and exploration behavior, a dramatic increase in forepaw and hindpaw diameters, radiological abnormalities, and maximal hyperalgesia; and (3) 11 weeks after the inoculation, a slight decrease in the number of Fos-LI neurons as compared to 3 weeks, but at 22 weeks, the number of Fos-LI neurons did not differ from control values. Interestingly, at this recovery stage, the hyperalgesia to mechanical pressure or to the foot-bend procedure was no longer observed. Fos-LI predominated in the neck of the dorsal horn (laminae V and VI, 55\% vs all the laminae) and to a lesser extent in the ventral horn ( $35 \%$ vs all the laminae), therefore contrasting the labeling observed following acute inflammation (Draisci and Iadarola, 1989; Menétrey et al., 1989; Noguchi et al., 1992), which is mainly located in the superficial laminae of the dorsal horn. Interestingly, Fos labeling in deep dorsal horn has been already reported after peripheral nerve injury (Basbaum et al., 1992; but see Williams et al., 1991; Herdegen et al., 1992).

On the basis of the above data, we chose to gauge the effects of pretreatment with diluted CFA, 3 weeks after inoculation of the disease. The number of Fos-LI neurons and their distribution within the spinal gray matter were remarkably similar to our initial study (Abbadie and Besson, 1992a). In contrast, in vaccinated rats, there was a clear decrease in the number of Fos-LI neurons $(13.5 \pm 5.2$ Fos-LI neurons per section vs 42.5 \pm 5.0 for vaccinated and arthritic, respectively). In the vaccinated group, the low number of neurons expressing c-fos could arise as a result of the induction of long-lasting physiopathological neural and immunological mechanisms, resulting in the animals being resistant to a second inoculation. Fos-LI was not completely blocked in the other control groups (except the "saline" group; mean of the three groups, 15 Fos-LI neurons). This labeling observed in the control groups that received the vehicle of the arthritogenic solution could have resulted from inflammation due to the emulsion (paraffin, Tween 80 , and saline). This finding is confirmed by the fact that in the fourth control group that received only saline throughout the study, the number of Fos-LI neurons was rather low $(3.3 \pm 2.0)$. It must also be emphasized that the number of Fos-LI neurons was significantly higher in the arthritic group as compared to the vaccinated group and to the four control groups, and there was no significant difference between the four groups of control.

A dramatic finding in our study is the fact that the reduction in the number of Fos-LI neurons is associated with a major decrease in pathological and behavioral signs of AIA disease. The reduction in inflammatory signs confirm and extend preliminary results on the severity of the disease (Waksman et al., 1960; Gery and Waksman, 1967). We extended these observations and considered the paw diameter and weight, which are indirect indicators of a chronic pain state (Chudler and Dong, 1983; Morton and Griffiths, 1985). In addition, the impairment of mobility that is at least partly a consequence of chronic pain states (Larsen and Arnt, 1985) was also determined. To explain these effects, it must be remembered that AIA in rats appears to result from an immunological reaction of the cellular type to disseminated constituents of the injected-killed bacteria (see references in Gery and Waksman, 1967). AIA has been shown to be prevented or reduced in rats that had received prior in- jection(s) of Mycobacterium by any routc, since certain antigens present on previously injected bacteria may induce tolerance to arthritogenic epitopes (Gery and Waksman, 1967). The critical Mycobacterium antigen contains an epitope cross-reactive with a self-antigen in joint cartilage, and administration of this cloned epitope antigen in joint cartilage in rats induces resistance to subsequent attempts to produce AIA (van Eden et al., 1988). Experimental evidence suggests that this autoimmune process involving $\mathrm{T}$-lymphocytes is responsible for the generation of AIA, since rats inoculated with a $\mathrm{T}$-cell line selected for its reactivity to the whole Mycobacterium present in CFA were fully protected against subsequent induction of the active AIA (Holoshitz et al., 1983). The pretreatment effect did not depend on nonspecific inhibition of inflammation but appeared to be due to either a production of specific tolerance or inhibition of sensitization by stimulating a response to competing antigens as proposed by Gery and Waksman (1967).

The decrease in inflammatory processes associated with the low number of Fos-LI neurons in the vaccinated group suggests that Fos-LI in spinal cord reflects some of the nociceptive signs associated with the disease. However, considering only the inflammatory signs is not sufficient enough to validate this assertion fully. Thus, we also considered the effects of vaccination on behavioral tests that are associated with nociccptive processes, which are in turn related to the extent of the arthritic disease (vocalization threshold to paw pressure, and to flexion or extension of the paw). However, it was not possible to perform a Fos-LI study and pain-related tests in the same animals (since it would not have been possible to distinguish between the Fos-LI induced by AIA and that induced by the stimulation; Abbadie and Besson, 1993). The scores of the vaccinated rats in pain-related tests clearly showed that they did not display hyperalgesia. The differences observed between the arthritic and the vaccinated group strongly indicate that the prevaccination procedure prevents chronic pain due to AIA.

The results obtained in the present study reinforce our previous observations that during the development of arthritis FosLI in the spinal cord may be a putative marker of chronic pain: the number of Fos-LI neurons is maximal when the pathological and behavioral signs of the disease are at their peak. In contrast, with a vaccination, the number of Fos-LI neurons was considerably lower when these signs were clearly reduced. In addition, we have previously shown that a 2 week chronic treatment with either aspirin or acetaminophen (paracetamol) prevents both the pathological signs associated with arthritis and Fos-LI in lumbar spinal cord (Abbadie and Besson, 1992b).

From a general point of view, these data are reminiscent of numerous previous investigations (see the introductory remarks) showing that various modalities of noxious stimulation, including acute inflammation, induce Fos-LI in the spinal cord. Briefly, acute stimulation induces Fos expression in both the superficial and deep laminae of the dorsal horn, while chronic arthritis induces Fos expression in the deep dorsal horn and in the ventral horn. These differences have already been discussed (Abbadie and Besson, 1992a). Interestingly, Fos is expressed in neurons that produce enkephalin (Noguchi et al., 1991) or dynorphin (Naranjo et al., 1991; Noguchi et al., 1991). Moreover, the Fos-Jun complex is able to bind to the AP-1-like sequence of enhancer region of the tyrosine hydroxylase (Gizang-Ginsberg and Ziff, 1990), preproenkephalin (Sonnenberg et al., 1989), and prodynorphin (Naranjo et al., 1991) genes. Thus, c-fos may play a crucial role in the regulatory mechanisms of the CNS in re- 
sponse to chronic pain diseases. In addition to the above-mentioned data, it is important to note that some of the Fos-LI cells observed in the present study may project to supraspinal levels. In fact, a certain number of Fos-LI cells following subcutaneous inflammation of the plantar hindpaw could be retrogradely labeled after administration of protein gold complex in various supraspinal regions (Menétrey et al., 1989). Thus, as already mentioned, the increase in the number of Fos-LI neurons during arthritis could indirectly reflect spontaneous chronic pain and hyperalgesia observed in these animals. In contrast, prevention of both disseminated inflammation and Fos expression by vaccination blocked the development of both hyperalgesia and spontaneous pain.

\section{References}

Abbadie C, Besson J-M (1992a) C-fos expression in rat lumbar spinal cord during the development of adjuvant-induced arthritis. Neuroscience 48:985-993.

Abbadic C, Besson J-M (1992b) Use of Fos-like immunoreactivity to study the effects of analgesic compounds in spinal cord neurons of polyarthritic rats. Soc Neurosci Abstr 18:429.2.

Abbadie C, Besson J-M (1993) C-fos expression in rat lumbar spinal cord following peripheral stimulation in adjuvant-induced arthritis and in normal rats. Brain Res 607:195-204.

Basbaum AI, Chi SI, Levine JD (1992) Peripheral and central contribution to the persistent expression of the C-fos proto-oncogene in spinal cord after peripheral nerve injury. In: Hyperalgesia and allodynia (Willis WD Jr, ed), pp 295-304. New York: Raven.

Besson JM, Chaouch A (1987) Peripheral and spinal mechanisms of nociception. Physiol Rev 67:67-186.

Besson JM, Guilbaud G (1988) The arthritic rat as a model of clinical pain? Amsterdam: Elsevier.

Bullitt E (1989) Induction of c-fos-like protein within the lumbar spinal cord and thalamus of the rat following peripheral stimulation. Brain Res 493:391-397.

Bullitt E (1990) Expression of c-fos-like protein as a marker for neuronal activity following noxious stimulation in the rat. J Comp Neurol 296:517-530.

Bullitt E (1991) Somatotopy of spinal nociceptive processing. J Comp Neurol 312:279-290.

Bullitt E, Lee CL, Light AR, Willcockson H (1992) The effect of stimulus duration on noxious-stimulus induced c-fos expression in the rodent spinal cord. Brain Res 580:172-179.

Butler SH, Weil-Fugazza J, Godefroy F, Besson J-M (1985) Reduction of arthritis and pain behaviour following chronic administration of amitryptiline or imipramine in rats with adjuvant-induced arthritis. Pain 23:159-175.

Calvino B, Crepon-Bernard M-O, Le Bars D (1987) Parallel clinical and behavioral studies of adjuvant-induced arthritis in the rat: possible relationship with "chronic pain." Behav Brain Res 24:11-29.

Calvino B, Couraud J-Y, Maillet S, Pradelles P, Besson J-M (1991a) Plasma and cerebrospinal fluid SP-like immunoreactivity in arthritic and Freund adjuvant pretreated rats. Soc Neurosci Abstr 17:478.8.

Calvino B, Maillet S, Pradelles P, Besson J-M, Couraud J-Y (1991b) Variations de l'immunoréactivité de type substance $P$ dans le plasma et le liquide c. . phalo-rachidien au cours de l'évolution de l'arthrite induite par adjuvant de Freund chez le rat, un modlele de douleur chronique. C R Acad Sci [III] 312:427-432.

Calvino B, Abbadie C, Besson JM (1993) Rats pretreated with diluted Freund adjuvant do not develop a chronic pain state associated with adjuvant induced arthritis: preclinical and c-Fos expression studies. 7th World Congress on Pain, abstract 113.

Cesselin F, Montastruc JL, Gros C, Bourgouin S, Hamon M (1980) Met-enkephalin levels and opiate receptors in the spinal cord of chronic suffering rats. Brain Res 191:289-293.

Chapman CR, Casey KL, Dubner R, Foley KM, Gracely RH, Reading AE (1985) Pain measurement: an overview. Pain 22:1-31.

Chudler EH, Dong WK (1983) Neuroma pain model: correlation of motor behaviour and body weight with autotomy in rats. Pain 17: 341-351.

Colpaert FC, De Witte P, Maroli AN, Awouters F, Niemegeers CJE,
Janssen PA (1980) Self-administration of the analgesic suprofen in arthritic rats: evidence of Mycobacterium butyricum-induced arthritis as an experimental model of chronic pain. Life Sci 27:921-928.

Colpaert FC, Bervoets KJW, Van den Hoogen RHWM (1987) Pharmacological analysis of hyperventilation in arthritic rats. Pain 30 : 243-258.

De Castro Costa M, De Sutter P, Gybels J, Van Hees J (1981) Adjuvant-induced arthritis in rats: a possible animal model of chronic pain. Pain 10:173-185.

Draisci G, Iadarola MJ (1989) Temporal analysis of increases in c-fos, preprodynorphin and preproenkephalin mRNAs in rat spinal cord. Mol Brain Res 6:31-37.

Gery I, Waksman BH (1967) Studies of the mechanism whereby adjuvant disease is suppressed in rats pretreated with mycobacteria. Int Arch Allergy 31:57-68.

Gizang-Ginsberg E, Ziff EB (1990) Nerve growth factor regulates tyrosine hydroxylase gene transcription through a nucleoprotein complex that contain c-Fos. Genes Dev 4:477-491.

Gogas KR, Presley RW, Levine JD, Basbaum AI (1991) The antinociceptive action of supraspinal opioids results from an increase in descending inhibitory control: correlation of nociceptive behavior and c-fos expression. Neuroscience 42:617-628.

Hammond DL, Presley RW, Gogas KR, Basbaum AI (1992) Morphine or U-50,488 suppresses Fos protein-like immunoreactivity in the spinal cord and nucleus tractus solitarii evoked by a noxious visceral stimulus in the rat. J Comp Neurol 315:244-253.

Herdegen T, Kovary K, Leah J, Bravo R (1991) Specific temporal and spatial distribution of JUN, FOS and KROX-24 proteins in spinal neurons following noxious transsynaptic stimulation. J Comp Neurol 313:178-191.

Herdegen T, Fiallosestrada CE, Schmid W, Bravo R, Zimmermann M (1992) The transcription factors c-JUN, JUN D and CREB, but not FOS and KROX-24, are differentially regulated in axotomized neurons following transection of rat sciatic nerve. Mol Brain Res 14:155165.

Holoshitz J, Naparstek Y, Ben-Nun A, Cohen IR (1983) Lines of T lymphocytes induce or vaccinate against autoimmune arthritis. Science 219:56-58.

Hsu S, Raine L, Fanger H (1981) Use of avidin-biotin-peroxidase complex (ABC) in immunoperoxidase techniques: a comparison between $\mathrm{ABC}$ and unlabelled antibody (PAP) procedures. J Histochem Cytochem 29:577-580.

Hunt SP, Pini $\Lambda$, Evan G (1987) Induction of $\mathrm{c}$-fos-like protein in spinal cord neurons following sensory stimulation. Nature 328:632634.

Hylden JLK, Noguchi K, Ruda MA (1992) Neonatal capsaicin treatment attenuates spinal Fos activation and dynorphin gene expression following peripheral tissue inflammation and hyperalgesia. J Neurosci 12:1716-1725.

Iadarola MJ, Brady LS, Draisci G, Dubner R (1988) Enhancement of dynorphin gene expression in spinal cord following experimental inflammation: stimulus specificity, behavioral parameters and opioid receptor binding. Pain 35:313-326.

Kuzuna S, Kawai K (1975) Evaluation of analgesic agents in rats with adjuvant arthritis. Chem Pharm Bull (Tokyo) 23:1184-1191.

Lantéri-Minet M, de Pommery J, Herdegen T, Weil-Fugazza J, Bravo R, Menétrey D (1992) Differential time-course and spatial expression of Fos, Jun and Krox-24 proteins in spinal cord of rats undergoing subacute or chronic somatic inflammation. J Comp Neurol 333:223235.

Larsen JJ, Arnt J (1985) Reduction in locomotor activity of arthritic rats as a parameter for chronic pain: effect of morphine, acetylsalicylic acid and citalopram. Acta Pharmacol Toxicol 57:345-351.

Leah JD, Sandkuhler J, Murashov A, Zimmermann M (1992) Potentiated expression of Fos protein in the rat spinal cord following bilateral noxious cutaneous stimulation. Neuroscience 48:525-532.

Lee J-H, Beitz AJ (1992) Electroacupuncture modifies the expression of c-fos in the spinal cord induced by noxious stimulation. Brain Res 577:80-91.

Menétrey D, Gannon A, Levine JD, Basbaum AI (1989) Expression of c-fos protein in interneurons and projection neurons of the rat spinal cord in response to noxious somatic, articular and visceral stimulation. J Comp Neurol 285:177-195.

Menétrey D, dc Pommcry J, Baimbridge KG, Thomasset M (1992) Calbindin-D28K (CaBP28k)-like immunoreactivity in ascending pro- 
jections. I. Trigeminal nucleus caudalis and dorsal vagal complex projections. Eur J Neurosci 4:61-69.

Molander C, Xu Q, Grant G (1984) The cytoarchitectonic organization of the spinal cord in the rat: I. The lower thoracic and lumbosacral cord. J Comp Neurol 230:133-141.

Morton DB, Griffiths PH (1985) Guidelines on the recognition of pain, distress and discomfort in experimental animals and an hypothesis for assessment. Vet Rec 116:431-436.

Naranjo JR, Mellström B, Achaval M, Sassone-Corsi P (1991) Molecular pathways of pain: Fos/Jun-mediated activation of a noncanonical AP-1 site in the prodynorphin gene. Neuron 6:607-617.

Noguchi K, Kowalski K, Traub R, Solodkin A, Iadarola MJ, Ruda MA (1991) Dynorphin expression and Fos-like immunoreactivity following inflammation induced hyperalgesia are colocalized in spinal cord neurons. Mol Brain Res 10:227-233.

Pearson CM, Wood FD (1959) Studies of polyarthritis and other lesions induced in rats by injection of mycobacterial adjuvant. I. General clinical and pathologic characteristics and some modifying factors. Arthritis Rheum 2:440-459.

Pearson CM, Waksman BH, Sharp JT (1961) Studies of polyarthritis and other lesions induced in rats by injection of mycobacterial adjuvant. J Exp Med 113:485-509.

Presley RW, Menétrey D, Levine JS, Basbaum AI (1990) Systemic morphine suppresses noxious stimulus-evoked Fos protein-like immunoreactivity in the rat spinal cord. J Neurosci 10:323-335.

Randall LO, Selitto J (1957) A method for measurement of analgesic activity on inflamed tissue. Anal Int Pharmacodyn 61:409-419.

Sonnenberg JL, Rauscher FJ III, Morgan JI, Curran T (1989) Regulation of proenkephalin by Fos and Jun. Science 246:1622-1625.

Tölle TR, Castro-Lopes JM, Coimbra A, Zieglgänsberger W (1990) Opiates modify induction of c-fos proto-oncogene in the spinal cord of the rat following noxious stimulation. Ncurosci Lett 111:46-51. van Eden W, Thole JER, van der Zee R, Noordzij A, van Embden JDA, Hensen EJ, Cohen IR (1988) Cloning of the mycobacterial epitope recognized by $\mathrm{T}$ lymphocytes in adjuvant arthritis. Nature 331:171-173.

Waksman BH, Pearson CM, Sharp JT (1960) Studies of arthritis and other lesions in rats by injection of mycobacterial adjuvant. II. Evidence that the disease is a disseminated immunologic response to exogenous antigen. $\mathrm{J}$ Immunol 85:403-417.

Williams S, Pini A, Evan G, Hunt SP (1989) Molecular events in the spinal cord following sensory stimulation. In: Processing of sensory information in the superficial dorsal horn of the spinal cord (Cervero F, Bennett J, Headley M, eds), pp 273-283. New York: Plenum.

Williams S, Evan GI, Hunt SP (1990) Changing patterns of c-fos induction in spinal neurons following thermal cutaneous stimulation in the rat. Neuroscience 36:73-81.

Williams S, Evan GI, Hunt SPF(1991) C-fos induction in the spinal cord after peripheral nerve lesion. Eur J Neurosci 3:887-894.

Winter CA, Kling PJ, Tocco DJ, Tanabe K (1979) Analgesic activity of diflusinal (MK-647; 5-(2,4-difluorophenyl) salicylic acid) in rats with hyperalgesia induced by Freund's adjuvant. J Pharmacol Exp Ther 211:625-678.

Wisden W, Errington ML, Williams S, Dunnett SB, Waters C, Hitchcock S, Evan G, Bliss TVP, Hunt SP (1990) Differential expression of immediate early genes in the hippocampus and spinal cord. Neuron 4:603-614.

Wood FD, Pearson CM (1962) Protection of rats against adjuvant arthritis by bacterial lipolysaccharides. Science 137:544-545.

Zimmermann M (1983) Ethical guidelines for investigations of experimental pain in conscious animals. Pain 16:109-110. 